\title{
A MEMS thermal shear stress sensor produced by a combination of substrate-free structures with anodic bonding technology
}

Yi Ou, Furong Qu, Guanya Wang, Mengyan Nie, Zhigang Li, Wen Ou, and Changqing Xie

Citation: Appl. Phys. Lett. 109, 023512 (2016); doi: 10.1063/1.4958842

View online: https://doi.org/10.1063/1.4958842

View Table of Contents: http://aip.scitation.org/toc/apl/109/2

Published by the American Institute of Physics

\section{Articles you may be interested in}

High temperature gradient micro-sensor for wall shear stress and flow direction measurements Applied Physics Letters 109, 241905 (2016); 10.1063/1.4972402

An integrated temperature-compensated flexible shear-stress sensor microarray with concentrated leading-wire Review of Scientific Instruments 87, 025001 (2016); 10.1063/1.4941067

Flow sensing by buckling monitoring of electrothermally actuated double-clamped micro beams Applied Physics Letters 109, 083503 (2016); 10.1063/1.4961582

A novel sacrificial-layer process based on anodic bonding and its application in an accelerometer AlP Advances 5, 041323 (2015); 10.1063/1.4907930

The fluctuating wall-shear stress and the velocity field in the viscous sublayer The Physics of Fluids 31, 1026 (1988); 10.1063/1.866783

Optimal thickness of silicon membranes to achieve maximum thermoelectric efficiency: A first principles study Applied Physics Letters 109, 053902 (2016); 10.1063/1.4960197

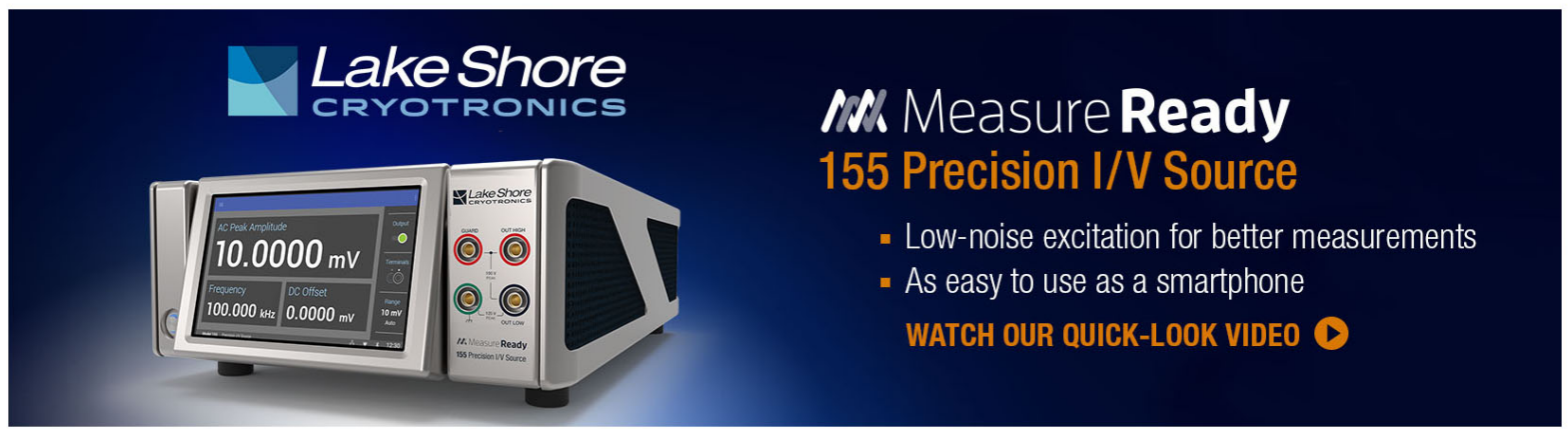




\title{
A MEMS thermal shear stress sensor produced by a combination of substrate-free structures with anodic bonding technology
}

\author{
Yi Ou, ${ }^{1}$ Furong Qu, ${ }^{1}$ Guanya Wang, ${ }^{1}$ Mengyan Nie, ${ }^{2}$ Zhigang Li, ${ }^{1}$ Wen Ou, ${ }^{1}$ \\ and Changqing Xie ${ }^{1, a)}$ \\ ${ }^{1}$ Key Laboratory of Microelectronic Devices and Integrated Technology, Institute of Microelectronics \\ of Chinese Academy of Sciences, Beijing 100029, People's Republic of China \\ ${ }^{2}$ National Centre for Advanced Tribology at Southampton (n CATS), Engineering Sciences, \\ University of Southampton, Southampton, SO17 1BJ, United Kingdom
}

(Received 12 April 2016; accepted 3 July 2016; published online 13 July 2016)

\begin{abstract}
By combining substrate-free structures with anodic bonding technology, we present a simple and efficient micro-electro-mechanical system (MEMS) thermal shear stress sensor. Significantly, the resulting depth of the vacuum cavity of the sensor is determined by the thickness of the silicon substrate at which $\mathrm{Si}$ is removed by the anisotropic wet etching process. Compared with the sensor based on a sacrificial layer technique, the proposed MEMS thermal shear-stress sensor exhibits dramatically improved sensitivity due to the much larger vacuum cavity depth. The fabricated MEMS thermal shear-stress sensor with a vacuum cavity depth as large as $525 \mu \mathrm{m}$ and a vacuum of $5 \times 10^{-2} \mathrm{~Pa}$ exhibits a sensitivity of $184.5 \mathrm{mV} / \mathrm{Pa}$ and a response time of $180 \mu \mathrm{s}$. We also experimentally demonstrate that the sensor power is indeed proportional to the 1/3-power of the applied shear stress. The substrate-free structures offer the ability to precisely measure the shear stress fluctuations in low speed turbulent boundary layer wind tunnels. Published by AIP Publishing.
\end{abstract}

[http://dx.doi.org/10.1063/1.4958842]

The wall shear stress, also known as skin friction, is defined as the force per unit area that a moving fluid exerts on a surface or wall. An accurate measurement of this parameter is of fundamental importance for the study of fluid dynamics on a solid boundary and the control strategies of aerodynamic flow. ${ }^{1-3}$ Several approaches have been developed to accomplish this goal. They can be categorized into two basic types, direct and indirect methods. ${ }^{4}$ Each of them has advantages and disadvantages. For the former such as Pitot tube, oil film interferometry and floating element sensors, they are able to measure the tangential force on the wall directly and are easy to use. However, they suffer from contamination by dust and moisture. For the latter such as thermal shear-stress sensors based on changes of physical measurands closely related to the shear stress, they are usually more robust than the former.

Inspired by the rapid development of micro-electromechanical system (MEMS) technology, MEMS-based thermal shear stress sensor has emerged as a promising candidate for replacing other conventional techniques in a better understanding of unsteady flow behaviors. This is not only because it can provide higher spatial and temporal resolution, and minimized flow interference, but also it has low cost and high reliability when batch fabricated using the silicon planar technique. ${ }^{5}$ Over the past two decades, continuous efforts have been devoted to develop various MEMS-based thermal shearstress sensors employing the sensing principle of heat transfer from a heated thin film element to the fluid flow. ${ }^{6,7}$ In particular, MEMS-based hot film sensors can accurately measure the fluctuating shear stress in a small region and are nonintrusive to the flow. However, they suffer from low frequency and

\footnotetext{
${ }^{\text {a) }}$ Author to whom correspondence should be addressed. Electronic mail: xiechangqing@ime.ac.cn
}

non-uniform phase responses. This is because they are very sensitive to parasitic losses of the supporting substrate. To overcome this problem, the combination of a hot film and a vacuum cavity structure was commonly used to reduce the total communication between the hot film sensor element and the underlying substrate. To create a thermal barrier which avoids heat to conduct away from the sensing element through the underlying substrate, a membrane with thickness in the micron range should be placed over the vacuum cavity.

The sensor performances depend sensitively on the volume of the fabricated vacuum cavity. Ideally, the depth of the vacuum cavity and the usable area of the membrane should be as large as the wafer thickness and the entire wafer area, respectively. Generally, this is obtained by using the so-called sacrificial layer technique from the front side of silicon substrate. ${ }^{8}$ Photolithography and reactive ion etching are needed to create access holes in the sacrificial layer, which are then removed to release free-standing structures, resulting in too much complexity and additional costs. Moreover, even though the modern sacrificial layer technique has led to diverse applications, the effective release of the sacrificial layer still remains a challenge due to the slow, diffusion-limited nature of chemical etch. The depth of the released cavity determined by the thickness of the sacrificial layer is usually less than $10 \mu \mathrm{m}$.

In this letter, we explore the feasibility of a simple and practical scheme for a MEMS-based thermal shear-stress sensor with very large vacuum cavity. Instead of using the sacrificial layer technique, we generate a substrate-free structure by conventional $\mathrm{KOH}$-based wet chemical etching from the bottom side of silicon substrate and combine it with anodic bonding technology to form a vacuum cavity. Such a vacuum cavity has a much larger volume than that fabricated 
by the conventional sacrificial layer technique, leading to an increased sensitivity to the MEMS-based thermal shearstress sensor. The dependences of the temperature change on the vacuum cavity depth were also investigated.

Our concept for the MEMS thermal shear stress sensor based on the combination of substrate-free structures and anodic bonding technology is schematically represented in Fig. 1(a). It consists of a vacuum cavity and a thermal resistor strip on the surface of the isolated thin film. The resistor strip is mounted into flow boundary layer while keeping the surface as flush as possible to reduce the influence of the flow field, where the air flow velocity changes from zero (at the wall) to the value of the mean stream velocity, as shown in Fig. 1(b). The resistor is heated by an electric current, and thus the heat lost in the air flow is dependent on the air flow velocity. The voltage across the thermal resistor can be measured with a read-out circuit. The measured values of the voltage are then used to calculate the shear stress associated with the mean flow velocity.

The total heat generated by the thermal resistor, $Q_{\text {total }}$,which stems from the heat stored in the resistor, the heat transferred to and stored on the insulation thin film, the heat transferred to and stored on the substrate and the convective heat transfer of air, can be expressed as ${ }^{9}$

$$
\begin{aligned}
Q_{\text {total }}= & h(\tau) A_{r}\left(T_{r}-T_{0}\right)+c_{r} m_{r} \frac{d T}{d t}+c_{f} m_{f} \frac{d T_{f}}{d t} \\
& +\frac{4 \lambda_{f} d_{f} l\left(T_{f}-T_{0}\right)}{L}
\end{aligned}
$$

where $l$ and $L$ are the lengths of the resistor and the isolation cavity side, respectively, $A$ and $d$ are the effective area and thickness of the resistor strip, respectively, $\lambda$ represents the thermal conductivity of the resistor material, $c$ denotes the specific heat, and $m$ is the mass. The subscripts $r$ and $f$ represent the heat transfer to the resistor and the insulation thin film, respectively.

The convective heat transfer coefficient $h(\tau)$ is a function of shear stress $\tau$ and can be written as ${ }^{10}$

(a)

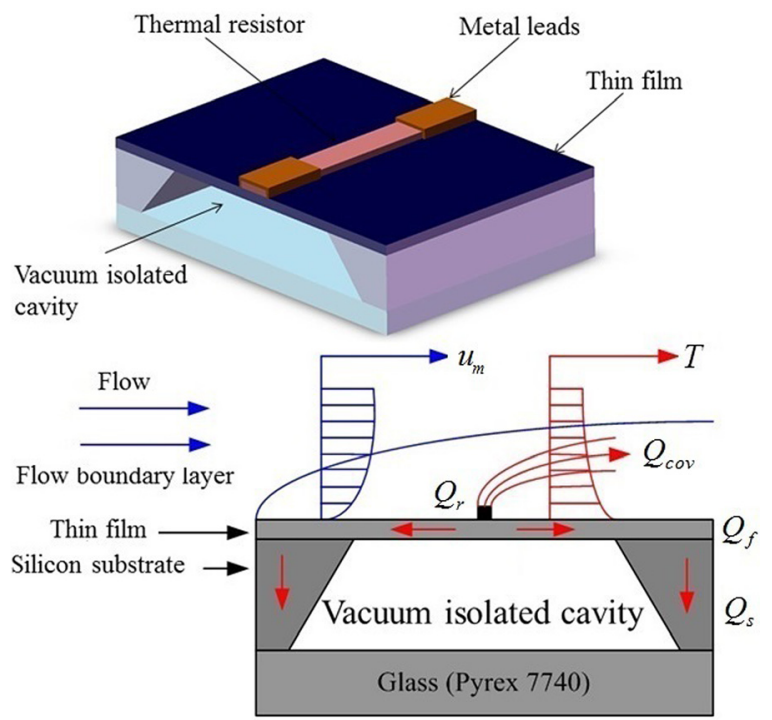

FIG. 1. (a) 3D view and (b) operation principle of the proposed shear stress sensor.

$$
h(\tau)=0.807\left(\frac{c_{a} \lambda_{a} \rho_{a} \tau}{w \mu}\right)^{1 / 3},
$$

where $w$ is the characteristic linear dimension (here defined as the width of the resistor strip), $w$ is the viscosity coefficient, and $\rho_{a}$ is the air density. The subscript $a$ represents the heat transfer to the air.

For simplicity and clarity, here a steady-state approximation is used, i.e., after the resistor is heated, the insulation thin film and the substrate have the same temperature change $(\Delta \mathrm{T})$. According to the so-called ohmic heating formula, and substituting Eq. (2) into Eq. (1), $Q_{\text {total }}$ is then given by

$$
\begin{aligned}
Q_{\text {total }}= & U^{2} / R_{s}=0.807\left(\frac{c_{a} \lambda_{a} \rho_{a} \tau}{w \mu}\right)^{1 / 3} \Delta T A_{r} \\
& +\frac{\lambda_{r} A_{r} \Delta T}{d_{r}}+\frac{\lambda_{f} A_{f} \Delta T}{d_{f}}+\frac{\lambda_{a} A_{f} \Delta T}{d}+\frac{4 \lambda_{f} d_{f} l \Delta T}{L},
\end{aligned}
$$

where $\mathrm{U}$ is the voltage across the resistor, $R_{S}$ is the thermal resistor and takes the form

$$
R_{s}=R_{0}\left[1+\alpha\left(T-T_{0}\right)\right],
$$

where $R_{0}$ and $T_{0}$ are the resistance and temperature under the ambient condition, respectively, and $\alpha$ is the temperature coefficient of resistance (TCR). In addition, the thermal response time is an important figure of merit for the sensor. By applying Laplace transformation to Eq. (3), we obtain

$$
t=\frac{c_{f} m_{f} d_{f}}{\lambda_{f} A}+\frac{c_{r} m_{r} d_{r}}{\lambda_{r} A}+\frac{c_{f} m_{f} L}{4 \lambda_{f} d_{f} l} .
$$

Here, platinum $(\mathrm{Pt})$ was selected as the thermal resistor material due to its high TCR value of $0.39 \%$. The temperature changes versus the vacuum cavity depth are plotted in Fig. 2 for the same input power of $0.02 \mathrm{~W}$. This figure clearly shows that the temperature change will become larger as the depth of the vacuum cavity increases. Furthermore, the temperature rise of the vacuum cavity is about $20^{\circ}$ higher than that of the air cavity, which means that the vacuum cavity is more desired for the reduction of the heat loss. For the sensor

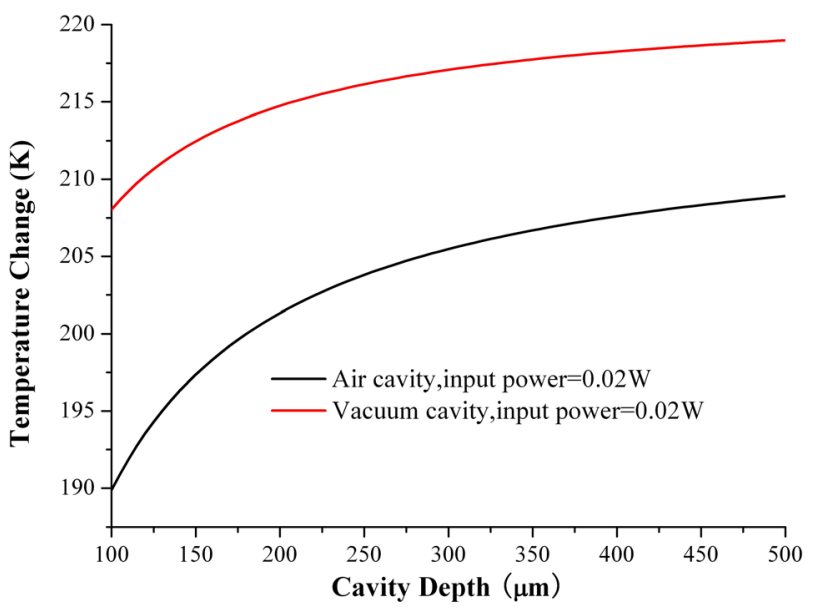

FIG. 2. Relationship between the temperature change and the vacuum cavity depth at the same input power. 
(a)

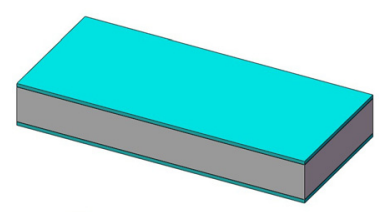

(b)

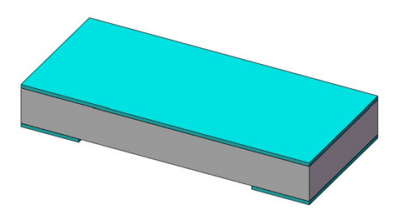

(c)

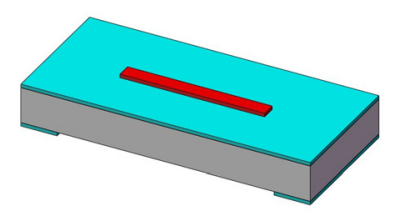

Silicon substrate

Glass (Pyrex 7740)

Aluminum leads

FIG. 3. Schematic of the major fabrication steps.

with a cavity side length of $200 \mu \mathrm{m}$, the thermal response time is calculated to be $153.8 \mu \mathrm{s}$.

As stated above, to avoid the complex sacrificial layer process, substrate-free structures were employed to form the isolated cavity with very large volume. Fig. 3 shows the major fabrication steps. The sensor fabrication started with a 4 in. (100) orientated p-type double-side-polished silicon with a thickness of $525 \mu \mathrm{m}$. Each sensor covers an area of about 3 square millimeters, and above 2500 sensors can be obtained per wafer. A SiNx layer with a thickness of $1.5 \mu \mathrm{m}$ was first deposited onto both sides of a silicon substrate by low pressure chemical vapor deposition [Fig. 3(a)]. Proximity optical lithography and reactive ion etching were then used to open a window on the back side of the silicon substrate [Fig. 3(b)]. A Pt resistor strip structure was then formed by the standard lift-off process with ultraviolet exposure of the photoresist on the front side of the silicon substrate [Fig. 3(c)]. Subsequently, aluminum leads with a thickness of $0.3 \mu \mathrm{m}$ were formed by another standard lift-off process on the front side of the silicon substrate [Fig. 3(d)]. It should be noted that a proper alignment is required to ensure that the Pt resistor strip and aluminum leads are positioned right during the ultraviolet exposures. The silicon exposed by the back side window was etched away using $\mathrm{KOH}$ aqueous solution, creating a SiNx membrane with a thickness of $1.5 \mu \mathrm{m}$ [Fig. 3(e)]. To protect the aluminumlead alloy structures during the anisotropic wet etching process, the front side of the substrate was covered by a glass plate and mounting adhesive (Crystalbond ${ }^{\mathrm{TM}}$ 509, Aremco Products Inc.). After wet etching the backside of the silicon substrate, the residual adhesive was removed by acetone. Finally, the silicon wafer was anodically bonded $\left(450{ }^{\circ} \mathrm{C}\right.$ for $60 \mathrm{~min}$ with a ramp rate of $30^{\circ} \mathrm{C} / \mathrm{min}$, a bonding voltage of $1500 \mathrm{~V}$ and a base pressure of $5 \times 10^{-2} \mathrm{~Pa}$ ) on a borosilicate glass wafer (Pyrex 7740), covering all of the isolated cavity [Fig. 3(f)].

The pressure of about $5 \times 10^{-2} \mathrm{~Pa}$ was obtained for the fabricated isolated cavity. Finally, the sensors were packaged in a standard dual-in-line package as shown in Fig. 4(a) and were assembled with a printed circuit board as shown in Fig. 4(b). The schematic diagram of the printed circuit board is illustrated in Fig. 4(c). Figs. 4(d) and 4(e) show the optical microscopy image and scanning electron microscopy (SEM) image of the fabricated sensor, respectively, clearly demonstrating that no $\mathrm{KOH}$ solution was found to corrode the front side of the aluminum-lead alloy structures.

To find the contribution of the cavity to the currentvoltage (I-V) characteristics, here we investigated three different types of sensors: (i) with vacuum isolation cavity $\left(5 \times 10^{-2} \mathrm{~Pa}\right)$; (ii) with air-filled cavity; and (iii) with no cavity. With a Keithley 4200-SCS semiconductor characterization system and a cascade RF-1 microwave probe station, the currents passing through the resistors were recorded at the same applied voltage. The I-V curve measurement results are shown in Fig. 5. At the same applied voltage, the current of the sensor with a vacuum cavity is smaller than those of the two other sensors, indicating that the sensor with a vacuum cavity has the largest resistance value. The I-V curve of the sensor with a vacuum cavity also exhibits a large nonlinearity, indicating that the vacuum cavity has a profound impact on the ohmic heating. For the sensor with no cavity, the $\mathrm{I}-\mathrm{V}$ characteristic curve is a linear function. This can be attributed to the fact that the heat is mainly transferred within (a)

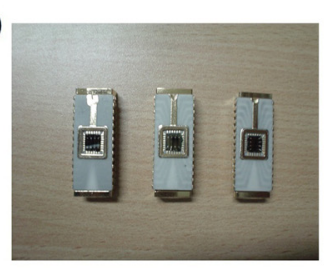

(c)

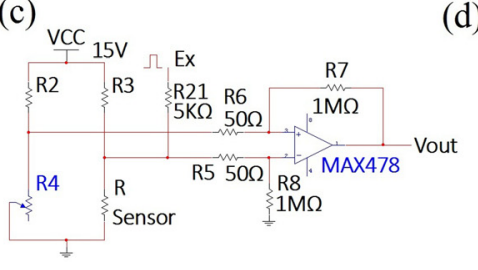

(d)

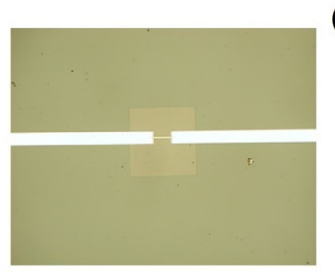

(b)

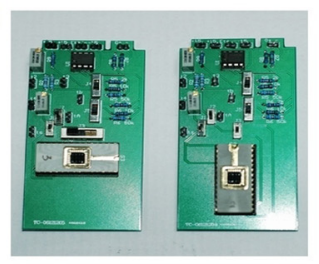

(e)

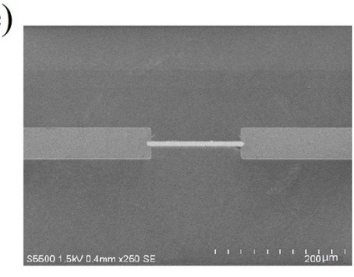

FIG. 4. (a) The packaged sensors. (b) The fully assembled sensors with test circuit boards. (c) Schematic diagram of the printed circuit board. (d) Top view image of the fabricated sensor. (e) SEM image of the fabricated sensor. 


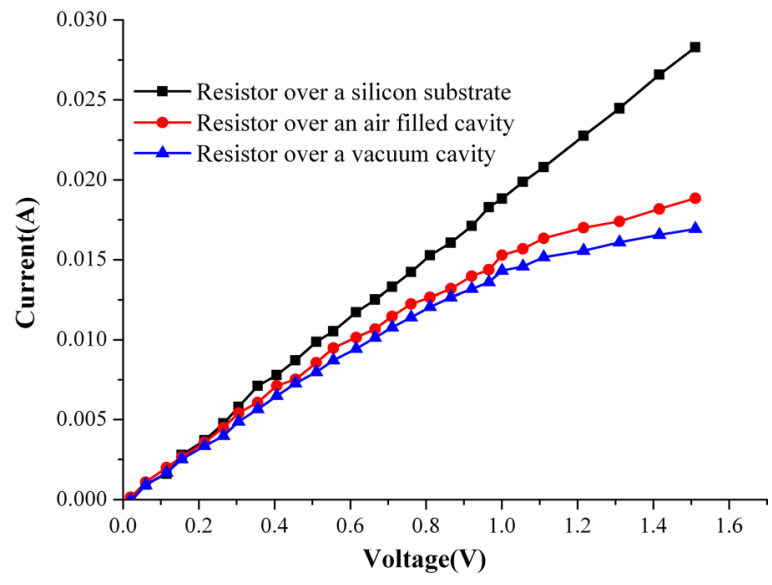

FIG. 5. I-V curves of three different types of fabricated sensors.

the thermal resistor placed on the silicon substrate. To further understand the effect of thermal isolation, here we define a factor $\theta$ affecting the thermal efficiency as $\theta=\mathrm{P} / \Delta \mathrm{T}$, where $\mathrm{P}$ is the input power determined by the equation $\mathrm{P}=\mathrm{IV}, \Delta T$ is the temperature rise in the resistor. The calculated $\theta$ values for these three different types of sensors with vacuum isolation cavity, with air-filled cavity, and with no cavity are $8.0 \times 10^{3}{ }^{\circ} \mathrm{C} / \mathrm{W}, 6.8 \times 10^{3}{ }^{\circ} \mathrm{C} / \mathrm{W}$, and $2.0 \times 10^{3}{ }^{\circ} \mathrm{C} / \mathrm{W}$, respectively. These results demonstrate that the sensor with vacuum isolation cavity offers the best thermal isolation. This is due to the fact that this sensor has a much larger vacuum cavity than those of the two other sensors.

Electronic test signals were also used in the determination of the time constant of the sensor. By inputting a square wave voltage signal into the Ex terminal of the readout circuit presented in Fig. 4(c), the thermal response time was measured to be $180 \mu \mathrm{s}$, as shown in Fig. 6, which is close to the calculated value of $153.8 \mu \mathrm{s}$. Before applying to a shear stress measurement, the MEMS thermal shear stress sensor calibration was performed based on an empirical relationship between the Reynolds number and the shear stress. The calibration experiment was carried out in a wind tunnel, where the velocity of air flowing can be adjusted by the level of a power supply. Fig. 7(a) shows the measured output voltage

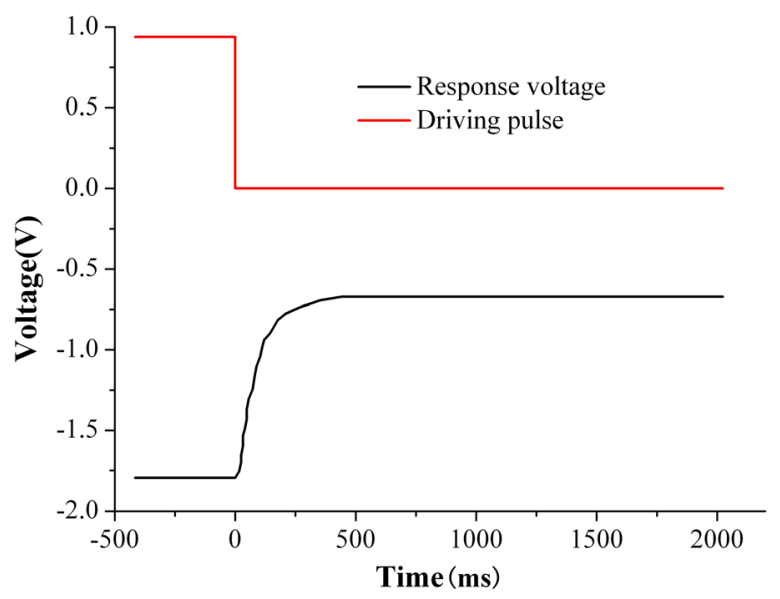

FIG. 6. The thermal response time measurement result of the sensor produced by a combination of substrate-free structures with anodic bonding technology.
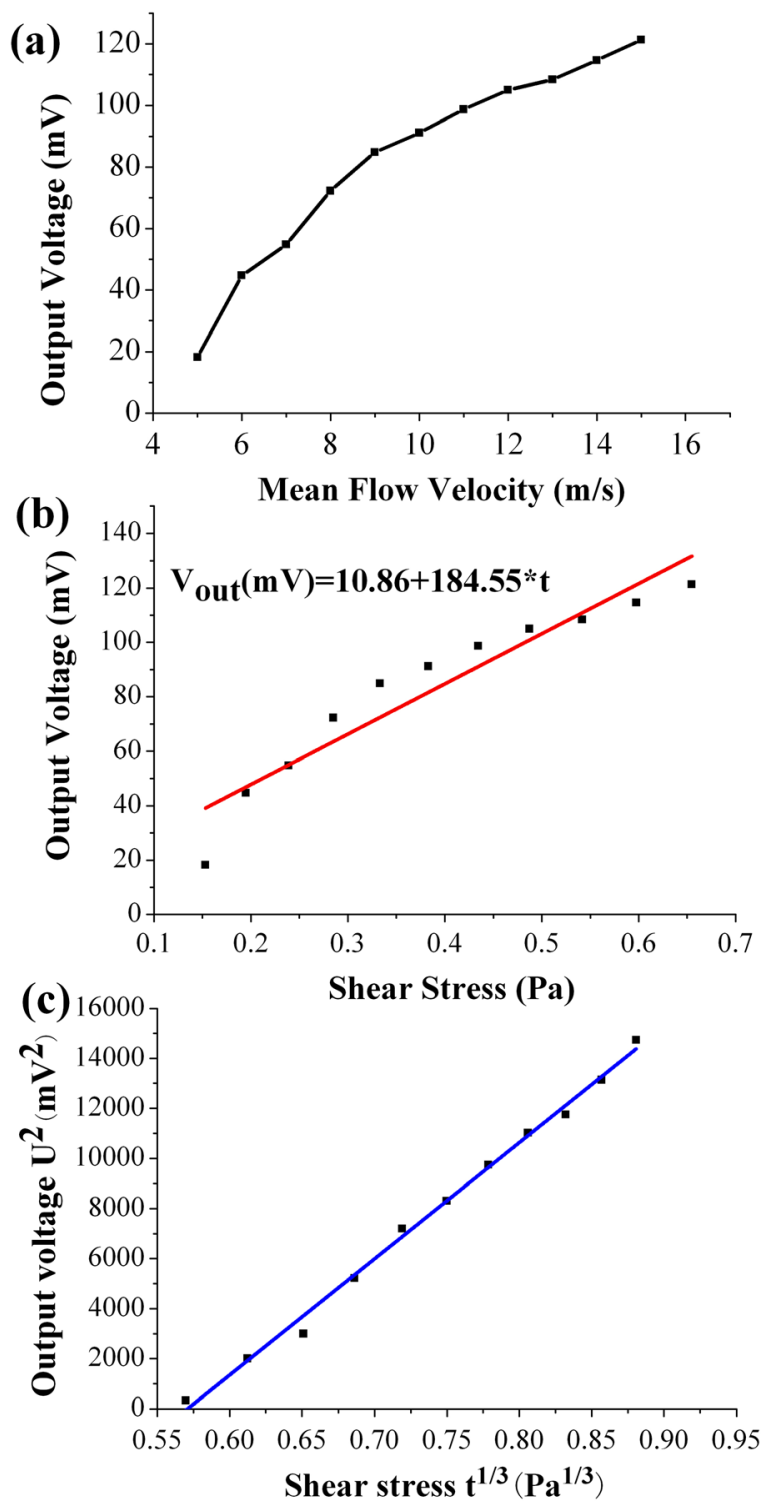

FIG. 7. (a) The measured output voltages of the fabricated sensor versus air mean flow velocity in the wind tunnel. (b) The calculated relationship between the measured output voltage and the applied shear stress. (c) The calculated relationship between the norm of output voltage and the cube root of the applied shear stress.

of the fabricated sensor with a vacuum cavity depth of $525 \mu \mathrm{m}$ versus mean airflow velocity ranging from 5 to $15 \mathrm{~m} / \mathrm{s}$. According to the fluid theory, the shear velocity $u$ is a function of the Reynolds number $R e$ and the mean airflow velocity $u_{m}$. The shear stress can be expressed as ${ }^{11}$

$$
\tau=0.332 R e^{-0.5} \rho u^{2}
$$

A relationship was established between the shear stress and the measured output voltage of the sensor with the vacuum cavity, as shown in Fig. 7(b). The sensitivity of the sensor is estimated to be $184.5 \mathrm{mV} / \mathrm{Pa}$. To gain further insight into the sensor behavior, we analyzed and fitted the data using the least square method. The dependence of the heating rate of the sensor on the applied shear stress in steady state is given by

$$
U^{2}=0.807 \tau^{1 / 3}+0.334
$$


Fig. 7(c) shows the relationship between the norm of output voltage and the cube root of the applied shear stress. One can observe that the experimental result is in good agreement with the classical theory that the sensor power is proportional to the $1 / 3$-power of the shear stress. ${ }^{12}$ Thus, Eq. (3) can be used to correctly predict the trend of our MEMS thermal shear-stress sensor operation.

In conclusion, we have demonstrated theoretically and experimentally a MEMS thermal shear stress sensor with very large vacuum cavity. The basic idea is to use a combination of substrate-free structures with anodic bonding technology, first to fabricate a substrate-free structure by anisotropic wet chemical etching of silicon, rather than the sacrificial layer technique, and then to form a vacuum cavity with anodic bonding technology. Thus, a vacuum cavity with a depth as large as the thickness of the silicon substrate used can be obtained in a controlled manner, paving the way for the realization of robust MEMS thermal shear stress sensor with dramatically improved sensitivity. A prototype MEMS thermal shear-stress sensor with a vacuum cavity depth as large as $525 \mu \mathrm{m}$ and a vacuum of $5 \times 10^{-2} \mathrm{~Pa}$ was fabricated, exhibiting a sensitivity of $184.5 \mathrm{mV} / \mathrm{Pa}$ and a response time of $180 \mu \mathrm{s}$. Furthermore, our experimental data also confirm that the sensor power is indeed proportional to the $1 / 3$-power of the applied shear stress. Such sensors should have great potential in applications such as the precise measurement of shear stress fluctuations for low speed turbulent boundary layer wind tunnels.

This work was supported by National Natural Science Foundation of China (Grant Nos. 61274119, 61306141, 61275170 and 51175535) and Research and Development of Major Research Equipment of Chinese Academy of Sciences (YZ201446).

${ }^{1}$ J. M. Ginder and L. C. Davis, Appl. Phys. Lett. 65(26), 3410 (1994).

${ }^{2}$ Z. X. Zhao, M. Shin, J. M. Gallman, and R. D. White, Sens. Actuators, A 205, 133 (2014).

${ }^{3}$ M. Shikida, Y. Yamazaki, K. Yoshikawa, and K. Sato, Sens. Actuators, A 189, 212 (2013).

${ }^{4}$ C. Liu, J.-B. Huang, Z. Zhu, F. Jiang, S. Tung, Y.-C. Tai, and C.-M. Ho, IEEE J. Microelectromech. Syst. 8(1), 90 (1999).

${ }^{5}$ M. Bulut Coskun, S. Moore, S. O. Reza Moheimani, A. Neild, and T. Alan, Appl. Phys. Lett. 104(15), 153502 (2014).

${ }^{6}$ L. Löfdahl and M. Gad-el-Hak, Meas. Sci. Technol. 10(8), 665 (1999).

${ }^{7}$ J. Miau, T. S. Leu, J. M. Yu, J. K. Tu, C. T. Wang, V. Lebiga, D. Mironov, A. Pak, V. Zinovyev, and K. M. Chung, Sens. Actuators, A 235, 1 (2015).

${ }^{8}$ B. J. Kim and E. Meng, J. Micromech. Microeng. 26(1), 013001 (2016).

${ }^{9}$ J. W. Naughton and M. Sheplak, Prog. Aerosp. Sci. 38(6), 515 (2002).

${ }^{10}$ J. B. Huang, S. Tung, C. M. Ho, C. Liu, and Y. C. Tai, IEEE Trans. Instrum. Meas. 45(2), 570 (1996).

${ }^{11}$ P. K. Kundu, I. M. Cohen, and D. R. Dowling, Fluid Mechanics (Academic Press, New York, 2011).

${ }^{12}$ R. J. Goldstein, Fluid Mechanics Measurements (Taylor \& Francis, New York, 1996). 